\title{
Impressum, Vol. 1, No. 5, 1973-74
}

S. Karger Verlag für Medizin und Naturwissenschaften GmbH, 8034 Germering, Postfach 2 Neurochirurgie

Anästhesie

Chirurgie

Biochemie

Pädiatrie

Innere Medizin

Anästhesie

Chirurgie

Anästhesie

Urologie

Anästhesie

Ernährungsphysiologie

Urologie

Herausgeber

Prof. Dr. H. Reissigl, W. Hofrat

Vorstand des Zentralinstituts für Bluttransfusion und Immuno-

logische Abteilung der Universitätskliniken Innsbruck, an der

Chirurgischen Universitätsklinik, Anichstraße 35, A-6020

Innsbruck

Prof. Dr. H. Grobecker

Zentrum für Pharmakologie, Abt. Ill, Johann-Wolfgang-

Goethe-Universität, Theodor-Stern-Kai 7, D-6000 Frankfurt/

Main 70

Prof. Dr. U. Henneberg

Geschäftsführender Direktor des Instituts für Anästhesiologie im Klinikum Steglitz der Freien

Universität Berlin, Hindenburg-damm 30, D-1 Berlin 45

Prof. Dr. H. G. Lasch

Direktor der Medizinischen Kliniken und Polikliniken der Justus-

Liebig-Universität, Klinikstraße 32b, D-63 Gießen

Wissenschaftlicher Beirat

E.H.Abel, Stade Verwaltung

Dr. W.Bayerl, Bonn Chirurgie

Prof. Dr. H. Bergmann, Linz Anästhesie

Dr. H. Boll, Hamm Anästhesie

Prof. Dr. C. Burri, Ulm Chirurgie

Prof. Dr. H. Busch, Hamburg Bluttransfusion

Prof. Dr. W. Dick, Ulm Anästhesie

Prof. Dr. R. Dohrmann, Berlin Chirurgie 
Prof. Dr. H. Egli, Bonn Bluttransfusion

Prof. Dr. M. Eggstein, Tubingen Innere Medizin

Prof. Dr. C. C. Ehrich, New York Bluttransfusion

Prof. D $\gamma$.H.FÖrster, FrankfurtBiochemie

Prof. Dr. V. Friedberg, MainzGynäkologie

Dr. P. Frank, Würzburg Pharmazie

Prof. Dr. H. E. Franz, Ulm Nephrologie

Prof. Dr. M. Gemperle, Genf Anästhesie

Prof. Dr. A. Hässig, Bern Hämatologie

Prof. Dr. O. Heidenreich, Aachen Pharmakologie

Prof. A. Heidland, Würzburg Innere Medizin

Dr. P. U.Heuckenkamp, München Innere Medizin

Prof. Dr. G. Hossli, Zurich Anästhesie

Prof. Dr. B. Hölscher, Berlin Chirurgie

Dr. K.F.Kopp, München Nephrologie

Prof. Dr. F. Krück, Homburg/Saar Innere Medizin

Dr. H. J. Kümmell, Melsungen Pharmazie

Priv. Doz. Dr. V. O. Lang, München Pädiatrie

Prof. Dr. A. Lindner, Wien Pharmakologie

Prof. Dr. A. Linke, Ludwigshafen Innere Medizin

Prof. Dr. P. Lundsgaard-Hansen Exp. Chirurgie

Prof. Dr. H. Lutz, Mannheim Anästhesie

Priv. Doz. Dr. J. Papenberg, Heidelberg Innere Medizin

Prof. Dr. H. W. Pia, Gießen

Priv. Doz. Dr. K. Peter, Mannheim

Prof. Dr. G. Rodewald, Hamburg

Prof. Dr. L. Róka, Giessen

Prof. Dr. E. Rossi, Bern

Prof. Dr. G. Schettler, Heidelberg

Dr. H. Schmidt, Frankfurt

Prof. Dr. I. Staib, Darmstadt

Prof. Dr. H. Stoeckel, Bonn

Prof. Dr. W. Vahlensieck, Bonn

Prof. Dr. K. Wiemers, Freiburg

Prof. Dr. A. Wretlind, Stockholm

Prof. Dr. M.Ziegler, Heidelberg

Prof. Dr. W. E. Zimmermann, Freiburg Chirurgie

Prof. Dr. R. X. Zittel, Ludwigshafen Chirurgie

Schriftleitung

Hans-Dieter Viering

Waldschmidstraße 8, D-8130 Starnberg am See

Tel.: 08151-6866

Herstellung: Werner Zuckschwerdt, Starnberg Satz und Druck: Buch- und Offsetdruckerei

Walter Biering KG 8 München 40

Erscheinungsweise 
$»$ Die Infusionstherapie« erscheint zweimonatlich; pro Jahr er-scheint 1 Band zu je 6 Heften; jedes Heft hat ungefähr 80 Seiten Inhalt. Jahrgang 1 deckt sich nicht mit dem Kalenderjahr. Er hat einen von der Regel abweichenden Umfang von 8 Heften, die in Abständen von 2 Monaten im Zeitraum Oktober 1973 bis Dezem-ber 1974erscheinen.

Abonnementspreis

Bezugspreis für Jahrgang 1, 1973/74 (8 Hefte) DM 92,-/US \$ 35,-/SFr 96,-- (Abonnenten, die bis Februar 1974 bislang nur 6 Hefte = DM 69,- belastet erhielten, erhalten für die Erweite-rung des Jahrganges um 2 Hefte eine Nachbelastung von DM 23,-). 1 Einzelheft kostet DM 14,-/ US \$ 4,50/SFr. 14,40, einschließlich MwSt., zuzüglich Postgebühren.

Der Abonnementspreis ist im voraus zahlbar. Die Lieferung der Zeitschrift läuft weiter, wenn sie nicht spätestens 4 Wochen vor Abschluß eines Bandes abbestellt wird.

Sonderhefte sind nur in beschränkter Auflage vorrätig. Der Preis eines Sonderhefts ist abhängig vom Umfang. Abonnenten erhalten die Sonderhefte zu einem um 20\% vom Ladenpreis ermäßigten Vorzugspreis.

Abonnements

Abonnementsbestellungen können bei jeder Buchhandlung, bei der Post oder direkt beim Verlag aufgegeben werden: Bundesrepublik Deutschland/ Österreich: S. Karger GmbH, Postfach 2, D8034 Germering/München, Tel.: 089-844021, Telex: 524865 D, Postscheckkonto: München 40080-807 Schweiz: S. Karger AG, Arnold-Böcklin-Straße 25, CH-4011 Basel, Tel.: 061390880, Telex $62652 \mathrm{CH}$

Frankreich: S. Karger S.A., 42bis, Boulevard de la Tour-Mau-bourg, F-75007 Paris

England: John Wiley \& Sons Ltd., Baffins Lane, Chichester USA: Albert J.Phiebig, Inc., P.O. Box 352, White Plains, New York, NY 10602

Anzeigen

MPV med. pharm. Verwaltungsgesellschaft mbH \& Co. KG, Schießstättstraße 14, Postfach 1547, D-8130 Starnberg, Telefon (08151)13299.

Gültig ist die Preisliste Nr. 2 vom 1. 3. 1974.

Der Gesamtauflage der Zeitschrift sind beigefügt:

eine Bestellkarte des Verlages S. Karger für die Zeitschrift, eine Beilage der Firma Fresenius Pharma, Bad Homburg, eine Beilage der Firma Becton Dickinson GmbH, Heidelberg. 